\title{
Selective Germline Genome Edited Pig Meninges Grafts for the Abdominal Wall Closure in Damage Control Surgery
}

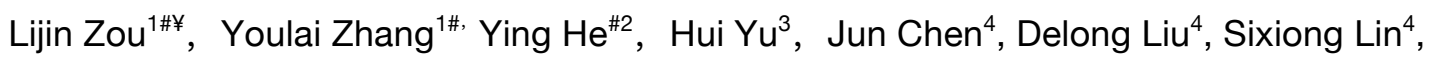
Manman $\mathrm{Gao}^{4}$, Gang Zhong ${ }^{5,6}$, Weicheng Lei ${ }^{7}$, Guangqian Zhou ${ }^{8^{*}}$, Xuenong Zou ${ }^{4^{*}}$, Kai $\mathrm{Li}^{9^{*}}$, Yin $\mathrm{Yu}^{5,6^{*}}$, , Gaofeng Zha ${ }^{10}$,Linxian $\mathrm{Li}^{10^{*} \neq}$, Yuanlin Zeng ${ }^{1 *}$,Jianfei Wang ${ }^{9 *}$, Gang Wang ${ }^{7,9^{*}}$

1. Burn Center, the First Affiliated Hospital of Nanchang University, Nanchang, Jiangxi,330006, People's Republic of China

2. Department of Infectious Diseases, the First Affiliated Hospital of Nanchang University, Nanchang, Jiangxi,330006, People's Republic of China

3. Department of Pathology, the First Affiliated Hospital of Nanchang University, Nanchang, Jiangxi,330006, People's Republic of China

4. Department of Surgery, Orthopedics Research Institute, Guangdong Provincial Key laboratory of Orthopedics and traumatology, the First Affiliated Hospital of Sun Yat-sen University, Guangzhou, Guangdong,510080, People's Republic of China

5. Shenzhen Institute of Synthetic Biology, Shenzhen Institutes of Advanced Technology, Chinese Academy of Sciences, Shenzhen, People's Republic of China

6. CAS Key Laboratory of Quantitative Engineering Biology, Shenzhen Institute of Advanced Technology, People's Republic of China

7. Zhuzao Biotechnology Inc, Shenzhen,581000, People's Republic of China

8. Department of Medical Cell Biology and Genetics, Guangdong Key Laboratory of Genome Stability \& Disease Prevention, Shenzhen Key Laboratory for Anti-ageing and Regenerative Medicine, Health Science Center, Shenzhen University, Shenzhen, 581000 People's Republic of China

9. Geneo Medicine Inc, Shenzhen,581000, People's Republic of China

10. Ming Wai Lau Centre for Reparative Medicine, Karolinska Institutet, Hong Kong,

\section{People's Republic of China}

\#: These authors contributed equally to this work

*: These authors contributed equally to this work

$¥$ : Correspondence should be addressed to L.Z.(zou.li.jin@hotmail.com), Y.H (cdyfyheying@hotmail.com), Y.Y.(yin.yu@siat.ac.cn), L.L.(linxian.li@ki.se), Y.Z.(zengyuanlin777@126.com), J.W.(jianfeiwang@geneomed.com), G.W.(gangwang@geneomed.com) 


\section{Abstract}

Reconstruction of abdominal wall defects is still a big challenge in surgery, especially where there is insufficient fascia muscular or excessive tension of the defects in emergency and life-threatening scenarios. Indeed, the concept of damage control surgery has been advanced in the management of both traumatic and nontraumatic surgical settings. The strategy requires abridged surgery and quick back to intensive care units (ICU) for aggressive resuscitation. In the damage control laparotomy, patients are left with open abdomen or provisional closure of the abdomen with a planned return to the operating room for definitive surgery. So far, various techniques have been utilized to achieve early temporary abdominal closure, but there is no clear consensus on the ideal method or material for abdominal wall reconstruction. We recently successfully created the selective germline genome-edited pig (SGGEP) and here we aimed to explore the feasibility of in vivo reconstruction of the abdominal wall in a rabbit model with SGGEP meninges grafts (SGGEP-MGs). Our result showed that the SGGEP-MGs could restore the integrity of the defect very well. After 7 weeks of engraftment, there was no sign of herniation observed, the grafts were re-vascularized, and the defects were well repaired. Histologically, the boundary between the graft and the host was very well integrated and there was no strong inflammatory response. Therefore, this kind of closure could help restore the fluid and electrolyte balance and to dampen systemic inflammatory response in damge control surgery while ADM graft failed to establish re-vascularization as the same as the SGGEP-MG. It is concluded that the meninges of SGGEP could serve as a high-quality alternative for restoring the integrity of the abdominal wall, especially for damage control surgery. 


\section{Introduction}

The congenital and acquired abdominal wall defects due to trauma, operation, tumour resection pose a formidable hurdle to the surgeons. Many different methods have been used for reconstruction but the optimal method is yet to be found. With the advent of prosthetic meshes the hernia recurrence rate is reduced $(1,2)$, but these implants could not establish re-vascularization and are not bioabsorbable. They not only could potentially cause the infection, chronic pain but also lead to other organ dysfunction, for example, intestine adherence or obstruction (3-10).

Notably, in the emergency and life-threatening scenario, the immediate goal is the closure of fascial defect as early as possible without triggering or exacerbating the lethal abdominal compartment syndrome (11-13). However, the management of these patients gives rise to a daunting challenge for surgeons. So far, techniques such as packing, mesh, and vacuum-assisted closure have been utilized to achieve early temporary abdominal closure. Nevertheless, there is no clear consensus on the ideal method or material for abdominal wall reconstruction. Each technique or material has their advantage and disadvantage $(14,15)$.

Very recently, we successfully established the selective germline genome edited pig (SGGEP) with a better immunological and molecular compatibility profile for humans (https://doi.org/10.1101/2020.01.20.912105). In this pig, three xenoantigens (Glycoprotein alpha-galactosyltransferase 1, GGTA; $\beta-1,4-\mathrm{N}$-acetyl-galactosaminyl 
transferase 2, B4GAL and, cytidine monophosphate- N- acetylneuraminic acid hydroxylase, $\mathrm{CAMH}$ ) which are well known to cause hyperacute rejection in xenotransplantation are knocked out, and additional eight human immune compatibility enhanced genes are knocked in, these genes encode a cluster human proteins including human complement system negative regulatory proteins (CD46, CD55, and CD59), human coagulation system negative regulatory proteins thrombomodulin (THBD), tissue factor pathway inhibitor (TFPI); CD39; macrophage negative regulatory proteins (human CD47); and natural killer cell negative regulatory human leukocyte antigen class I histocompatibility antigen, alpha chain E (HLA-E). When SGGEP skin was engrafted to NHP, up to 25 days' graft survival was observed, thus SGGEP skin grafts demonstrated the long immunological tolerance in NHP (https://doi.org/10.1101/2020.01.20.912105).

In the present study, we aimed to extend the utility for our SGGEP, we set out to explore the feasibility of in vivo reconstruction of the abdominal wall in a rabbit model using SGGEP meninges grafts (SGGEP-MG). Our results indicated that the SGGEP-MGs could repair the abdominal wall defect well by establishing revascularization and restoring the integrity of defected abdominal wall after 7 weeks engraftment while the pig acelluar Derm Matrix(ADM) grafts failed to repair the defects. Therefore, SGGEP-MG can be considered as a high-quality alternative, specially for the temporary closure of fascial defect techniques in the damage control surgery. This encouraging data offers the justification and a conceptual framework for ongoing NHP preclinical safety and efficacious assessment and potential future clinical applications. 


\section{Results}

The clinical gross appearance of the grafts was assessed every 7 days after transplantation. The post-operation day 49 was designed as the endpoint in the experiment group while in the control group, the post-operation day 35 was set as the endpoint. It was observed that the SGGEP-MG repaired abdominal wall defects well, it offered good healing for the defect with collagen fibrils-rich environment (Figure $2 \mathrm{~d}, \mathrm{e}$, f). There was no sign of herniation throughout the experiments observed. Importantly, the grafts were-vascularized and integrated with surrounding tissues very well, there was no strong inflammation response (Figurele,f,g,h) throughout the whole course of the study. The histological analysis confirmed these observations with minor inflammatory cell cells and rich of collagen in both junctional and central regions in the grafts (Figure $2 \mathrm{~d}, \mathrm{e}$, f). The collagen fibres were still observed by 7 weeks after transplantation and highdensity vessels were evidenced (Figure $2 \mathrm{~d}, \mathrm{e}, \mathrm{f}$ ).

In the control group, ADM grafts yielded quite different graft-host healing responses although there was also no sign of herniation observed. The boundary between the grafthost was clear both at clinical observation and histological analysis (Figure $1 \mathrm{a}, \mathrm{b}, \mathrm{c}, \mathrm{d}$, Figure $2 \mathrm{a}, \mathrm{b}, \mathrm{c}$ ) indicating that the grafts were not re-vascularized and integrated with neighbouring host tissues. The HE staining indicated that though the ADM could provide the temporary cover for the defect but failed to repair the abdominal wall defect by establishing revascularization and restoring the integrity as quickly as the SGGEP group. 


\section{Materials \& Methods:}

\section{Animals}

All studies were approved by the First Affiliated Hospital of Nanchang University Institutional Animal Care and Use Committee (IACUC) and performed in accordance with the Guidelines for the Humane Treatment of Laboratory Animals (2006) issued by the Chinese Government.

(http://www.most.gov.cn/fggw/zfwj/zfwj2006/200609/t20060930_54389.htm.

Japanese white rabbits $(2.5 \pm 0.5 \mathrm{~kg}, 6$-month-old, male; Longping Company, Nanchang, China) and SGGEP ( 3 months old) were used in these studies.

\section{SGGEP meninges grafts xenotransplantation in a rabbit peritoneum defect model.}

Swine donors were anesthetized with $2.5 \mathrm{mg} / \mathrm{kg}$ diazepam IM and $10 \mathrm{mg} / \mathrm{kg}$ ketamine IM. Then, endotracheal intubation was performed for anesthesia ( $2 \%$ isoflurane and oxygen). The head was shaved using clippers and then disinfected with Povidone-Iodine and rubbed with $70 \%$ ethanol, the skull was opened, $5 \times 5 \mathrm{~cm}$ cerebral dura grafts were harvested, the grafts for immediate use were immersed in saline and stored at $4{ }^{\circ} \mathrm{C}$. The pigs were all euthanized by an overdose of anesthetics.

The rabbits were anesthetized with $10 \%$ chloral hydrate $(4 \mathrm{ml} / \mathrm{kg})$ through intraperitoneal injections. The animals were divided into two groups. The experimental group $(n=7)$ were received SGGEP cerebral dura grafts. The control group $(n=5)$ were received ADM (acellular dermal matrix) grafts. A longitudinal skin incision was made and abdominal 
wall muscle was dissected bluntly. A $5 \times 5 \mathrm{~cm}$ peritoneal defect was made carefully by the ophthalmic scissor. For the experimental group, SGGEP-MGs were implanted to the defect site appropriately to close the defect while ADM was implanted as a control. The grafts were sutured to rabbit abdominal defect with 3-0 Ethilon by interrupted sutures. Pressure bandaging was applied to protect the incisional site. Ceftriaxone sodium $(80 \mathrm{mg} / \mathrm{kg}) \quad$ IV was administered once before and once after the operation. All rabbits were individually housed in a cage after the surgery. The graft clinical assessment was made as indicated time points to analyze the graft-host healing effects.

\section{Histopathology analysis}

After the engraftments, the samples were collected on different days. The samples were fixed in $4 \%$ paraformaldehyde and embedded in paraffin. Sections $(5 \mu \mathrm{m})$ were cut, mounted on the slide, and stained with hematoxylin and eosin (HE). The HE sections were viewed under x 100 magnification, photos were taken for analysis. 


\section{Discussion}

To close the abdominal wall, various techniques have been used for the past century for different etiology, location and degree (layers implicated) of the defects. Some utilized the mesh (16-18), the others used the flaps (19-21). Recent new procedures with better results were reported (22-24). However, each technique has its advantage and disadvantage. Ideally, after implantation of the mesh, several key host processes should occur, including the acute inflammatory response, followed by the migration of mononuclear cells to the site of implantation. After cellular penetration of the material, new blood vessel proliferation (neovascularization) extends through the thickness of the matrix. Concurrently, mononuclear cells (macrophages) secrete cytokines and other signalling molecules, resulting in the recruitment of fibroblasts to the region, deposition of endogenous collagen, and resorption of the mesh. These processes are considered integral to biologic mesh integration and remodelling (25).

With the aim of obtaining a tension-free closure of the abdominal wall defects, synthetic materials are often utilized to restore missing fascial tissues and strengthen the healing. But these nonabsorbable materials may cause complications, for instance, infections, chronic pain, erosions, and fistula formation, in particular when they are set directly over the viscera. While other materials are available, but each has its own pros and cons (2628). 
Especially, the damage control surgery practice is one of the greatest progress in recent decades and has established as a common procedure in both traumatic and general surgery. One of the damage control surgery purposes is the closure of the fascial defect as quickly as is clinically possible without rising the intraabdominal pressure during the initial hospitalization. Quite a few techniques have been reported workable in helping to increase the primary abdominal fascial closure rate. However, currently, high-quality comparative data is rare (15).

Biological materials have an advantage over these nonabsorbable and no blood vessel materials in view of maintaining physiological homeostasis. Very recently we establish the SGGEP with enhanced human compatibility (https://doi.org/10.1101/2020.01.20.912105). We aimed to evaluate if SGGEP MGs, rich in collagen and strong tensile strength, could serve as a better alternative. Moreover, we would like to assess if it could fulfill the re-vascularization which is a key to maintain the water and electrolyte balance and to combat the infection in the damge control surgery.

Indeed, in the present study, after implantation into abdominal wall defect, SGGEP MGs were observed to be re-vascularized, maintain its structural integrity and the boundary between the graft and the surrounding tissues was integrated indicating a good host -graft environment. While the ADM graft did not yield a good host-graft interaction and the boundary between the graft and host was clearly demarcated throughout the experiment course. Considering the effective repair of abdominal wall defects relying on the early reestablishment of vascular vessels (29), the SGGEP graft had a very satisfying result. 
As it is well known that an ideal graft should have characteristics such as strong, inert, resistant to infection, low recurrence rate, high quality of life and good value. Ongoing advancements in techniques and technology will continue to help surgeons tailor the graft to the clinical scenario(30). Therefore, it can be concluded that the SGGEP meninges graft is more effective for managing abdominal wall defects than ADM. It has the potential to be widely applied to deal with abdominal wall defects, particularly in emergency or infection situations. This study points out that the SGGEP meninges graft is effective and safe for the reconstruction of abdominal wall defects. SGGEP meninges implantation is a feasible and reproducible technique providing a large and functional covering of abdominal wall defect. The SGGEP meninges can be cryopreserved, stored for emergency events, and transported worldwide for global use (Figure 3). Further NHP pre-clinical investigations are necessary to assess the practical validity of this concept.

\section{Acknowledgements}

L.Z., Y.Z. was supported by the grant Natural Science Foundation of China(NSFC 81660364 and NSFC 81760343 ).X.Z. was funded by grants Natural Science Foundation of Guangdong Province (2017A030308004) and Natural Science Foundation of Guangzhou City (201804020011). G.Z. was supported by the National Key R\&D Program of China (2017FA105202) and Shenzhen Scientific \& Innovation Committee (GJHZ20180928155604671),Y.H. was funded by Health and Family Planning Project of Jiangxi Province(No. 20191025).

\section{Competing Financial Interests}

J.W., G.W., K.L. are inventors on a patent filed by Geneo Medicine Inc using the technology described in this paper. J.W, G.W, K.L., are employees of Geneo Medicine Inc, G.W.,W.L is an employee of Zhuzao Biotechology, Inc. 


\section{References:}

1. Burger JW, Luijendijk RW, Hop WC, Halm JA, Verdaasdonk EG, and Jeekel J. Long-term follow-up of a randomized controlled trial of suture versus mesh repair of incisional hernia. Ann Surg. 2004;240(4):578-83; discussion 83-5.

2. Luijendijk RW, Hop WC, van den Tol MP, de Lange DC, Braaksma MM, JN IJ, et al. A comparison of suture repair with mesh repair for incisional hernia. $N$ Engl J Med. 2000;343(6):392-8.

3. Aasvang E, and Kehlet H. Chronic postoperative pain: the case of inguinal herniorrhaphy. Br J Anaesth. 2005;95(1):69-76.

4. Engelsman AF, van der Mei HC, Ploeg RJ, and Busscher HJ. The phenomenon of infection with abdominal wall reconstruction. Biomaterials. 2007;28(14):2314-27.

5. Mathes SJ, Steinwald PM, Foster RD, Hoffman WY, and Anthony JP. Complex abdominal wall reconstruction: a comparison of flap and mesh closure. Ann Surg. 2000;232(4):586-96.

6. Falagas ME, and Kasiakou SK. Mesh-related infections after hernia repair surgery. Clin Microbiol Infect. 2005;11(1):3-8.

7. Abdollahi A, Maddah GH, Mehrabi BM, Jangjoo A, Forghani MN, and Sharbaf N. Prosthetic incisional hernioplasty: clinical experience with 354 cases. Hernia. 2010;14(6):569-73.

8. Jacob BP, Hogle NJ, Durak E, Kim T, and Fowler DL. Tissue ingrowth and bowel adhesion formation in an animal comparative study: polypropylene versus Proceed versus Parietex Composite. Surg Endosc. 2007;21(4):629-33.

9. Leber GE, Garb JL, Alexander AI, and Reed WP. Long-term complications associated with prosthetic repair of incisional hernias. Arch Surg. 1998;133(4):378-82.

10. Shin D, Lipshultz LI, Goldstein M, Barme GA, Fuchs EF, Nagler HM, et al. Herniorrhaphy with polypropylene mesh causing inguinal vasal obstruction: a preventable cause of obstructive azoospermia. Ann Surg. 2005;241(4):553-8.

11. Rotondo MF, Schwab CW, McGonigal MD, Phillips GR, 3rd, Fruchterman TM, Kauder DR, et al. 'Damage control': an approach for improved survival in exsanguinating penetrating abdominal injury. J Trauma. 1993;35(3):375-82; discussion 82-3.

12. Burch JM, Ortiz VB, Richardson RJ, Martin RR, Mattox KL, and Jordan GL, Jr. Abbreviated laparotomy and planned reoperation for critically injured patients. Ann Surg. 1992;215(5):476-83; discussion 83-4.

13. Jensen SD, and Cotton BA. Damage control laparotomy in trauma. Br J Surg. 2017;104(8):959-61.

14. Germanos S, Gourgiotis S, Villias C, Bertucci M, Dimopoulos N, and Salemis N. Damage control surgery in the abdomen: an approach for the management of severe injured patients. Int J Surg. 2008;6(3):246-52.

15. Huang Q, Li J, and Lau WY. Techniques for Abdominal Wall Closure after Damage Control Laparotomy: From Temporary Abdominal Closure to Early/Delayed Fascial Closure-A Review. Gastroenterol Res Pract. 2016;2016:2073260. 
16. Usher FC. A new plastic prosthesis for repairing tissue defects of the chest and abdominal wall. Am J Surg. 1959;97(5):629-33.

17. Jacobs E, Blaisdell FW, and Hall AD. Use of knitted marlex mesh in the repair of ventral hernias. Am J Surg. 1965;110(6):897-902.

18. Gilsdorf RB, and Shea MM. Repair of massive septic abdominal wall defects with Marlex mesh. Am J Surg. 1975;130(6):634-8.

19. Pridgen JE, and Tennison CW. Reconstruction of the entire anterior abdominal wall in the presence of postradiation changes. Am J Surg. 1956;92(1):54-6.

20. Nahai F, Silverton JS, Hill HL, and Vasconez LO. The tensor fascia lata musculocutaneous flap. Ann Plast Surg. 1978;1(4):372-9.

21. Bostwick J, 3rd, Hill HL, and Nahai F. Repairs in the lower abdomen, groin, or perineum with myocutaneous or omental flaps. Plast Reconstr Surg. 1979;63(2):186-94.

22. Shestak KC, Edington HJ, and Johnson RR. The separation of anatomic components technique for the reconstruction of massive midline abdominal wall defects: anatomy, surgical technique, applications, and limitations revisited. Plast Reconstr Surg. 2000;105(2):731-8; quiz 9.

23. Senghaas A, Kremer T, Schmidt VJ, Harhaus L, Hirche C, Kneser U, et al. Sliding free transverse rectus abdominis myocutaneous flap for closure of a massive abdominal wall defect: A case report. Microsurgery. 2019;39(2):174-7.

24. Chen Q, Liu Q, Suo Y, and Xie Q. A new surgical treatment for abdominal wall defects: A vascularized ribs-pleural transfer technique that can be used with or without a thoracic umbilical flap a case report. Medicine (Baltimore). 2018;97(9):e9993.

25. De Silva GS, Krpata DM, Gao Y, Criss CN, Anderson JM, Soltanian HT, et al. Lack of identifiable biologic behavior in a series of porcine mesh explants.

Surgery. 2014;156(1):183-9.

26. Patton JH, Jr., Berry S, and Kralovich KA. Use of human acellular dermal matrix in complex and contaminated abdominal wall reconstructions. Am J Surg. 2007;193(3):360-3; discussion 3.

27. Clarke KM, Lantz GC, Salisbury SK, Badylak SF, Hiles MC, and Voytik SL. Intestine submucosa and polypropylene mesh for abdominal wall repair in dogs. $J$ Surg Res. 1996;60(1):107-14.

28. Soiderer EE, Lantz GC, Kazacos EA, Hodde JP, and Wiegand RE. Morphologic study of three collagen materials for body wall repair. J Surg Res. 2004;118(2):161-75.

29. Bellows CF, Alder A, and Helton WS. Abdominal wall reconstruction using biological tissue grafts: present status and future opportunities. Expert Rev Med Devices. 2006;3(5):657-75.

30. Abid S, and El-Hayek K. Which mesh or graft? Prosthetic devices for abdominal wall reconstruction. Br J Hosp Med (Lond). 2016;77(3):157-8, 9-61. 
a

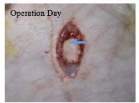

e

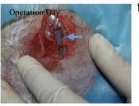

b

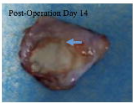

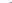
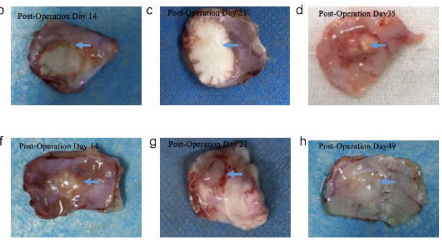

Fizure 1 The respective gross appearanec picture were showa after Selective (icrmline Genome Fditcd Pig meninges grafss(SGGFP-MG) to rabbit perituneurn xenotransplantatiun(a) Pig ADM(acellular dermel mairiz) grafl on the operal ku Day,(b) Pig ADM graft on the post-opocation Day 14, (c) Pig ADM grafl on the post-operation Day21, (d) Pig ADM grafl on the post-opcration

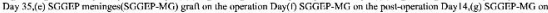
the post-operation Dyy21, h) SC,GIP-MG on the poxi-operation Day49. 


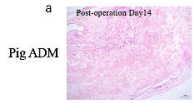

b

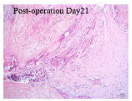

c

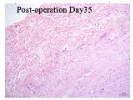

d

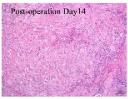

e

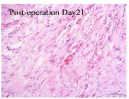

f

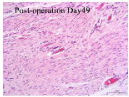

Figure 2 The respective histology HE staining images are skown from biopsies taken on after Selective Germilime Genome Edited Pig meninges grafts(SGGEP-MG) to rabbit peritoncum xenotransplantation (a) Pig ADM graft on the post-operation Day 14, (b) $P_{\mathrm{Lg}}$ ADM graft on the post-operation Day21, (c) Pig ADM graft on the post-operation Day 35,(c) SGCEP-MG on the post-operation Day 14,(e) SGGEP-MG on post-operation Day21,(f) SGGEP-MG on the post-operation Day 49 

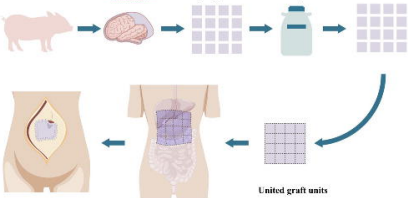

United graft units

Ckosure of abouninal wall wite united graff units esperially in damage cout rol surgery
Clesure of abdeninal wall with united graft enits

SGCEY : selective gerullue ger.one edited pig 